\title{
The Survival Processing Effect in Recall Memory: Survival and Planning
} Leisha A. Colyn \& Richard B. Anderson

Bowling Green State University

Research has demonstrated that processing information in a survival-related context enhances true recall ([1]). It also, however, increases false recall relative to other memory strategies and information processing contexts (e.g., contextually-rich narratives such as moving, and non-contextually rich semantic tasks such as pleasantness processing ([2])). This robust effect is known as the survival-processing effect.

Nairne and colleagues ([1]) suggest that survival may serve as a special domain of information processing that uniquely promotes true and false recollections. In contrast, Klein, Robertson, and Delton ([3, 4]) suggest that planning processes inherent to the survival processing task may explain the effect.

The current research examines the effects of survival salience and planning processing on true and false recall memory using the typical survival processing narrative, two non-survival, contextually-rich planning narratives, and a pleasantness control task.

If survival salience uniquely promotes true and false recollections then the survival task should produce greater true and false recall than the non-survival planning tasks. However, if planning explains the survival processing effect, then all planning tasks should produce greater true and false recall than the pleasantness processing task.

\section{Method}

In line with previous research on the survival processing effect, we used an incidental learning task that included learning, retention, and recall phases. This experiment was designed as a single-factor, between-participants design, with four groups. True and false recall were measured.

\section{Participants}

Two hundred and thirty-one undergraduate students (75 male, 155 female, 1 gender-unreported) participated in this study. Thirty-one percent of participants were university freshman, 19\% were sophomores, 9\% were juniors, $41 \%$ were seniors, and $<1 \%$ were either a college graduate or a graduate student.

Most participants self-identified as Caucasian (61\%), but the sample was considerably diverse and contained people identifying as AsianAmerican/Pacific Islander (14\%), African-American (13\%), Hispanic/Latino (6\%), and "Other" (7\%). 
Participants were randomly assigned to an encoding processing task. Twenty-four percent $(n=56)$ were assigned to the Grasslands Survival processing task, 26\% $(n=59)$ were assigned to the Grasslands Photo processing task (i.e., grasslands-survival-with-planning), $25 \%(n=58)$ were assigned to the Dinner Party processing task (i.e., non-grasslands-planning-without-survival), and $25 \%(n=58)$ were assigned to the Pleasantness processing task.

\section{Materials}

Three components were varied across narrative tasks: survival salience, grasslands context, and planning. See Table 1 for the characteristics of each processing task.

Table 1. Characteristics of the information processing tasks.

\begin{tabular}{|l|c|c|c|c|}
\hline & Survival & Grasslands & Planning & Narrative \\
\hline Grasslands Survival & Yes & Yes & Yes & Yes \\
\hline Grasslands Photo & No & Yes & Yes & Yes \\
\hline Dinner Party & No & No & Yes & Yes \\
\hline Pleasantness & No & No & No & No \\
\hline
\end{tabular}

The narrative tasks were as follows:

"Imagine that you are: (A) going to be stranded in the grasslands without any basic survival materials; (B) going to visit a grassland; (C) planning a dinner party for the weekend. You'll need to: (A) find steady supplies of food and water and protect yourself from predators; (B) take photographs of the geography, vegetation, and wildlife; (C) go to the store to purchase food, drinks, and other party supplies. Because you are not sure of the guests' food preferences, you plan on purchasing a variety of different things.

"Pay attention to all of the feelings and thoughts that go through your mind as you think about and plan for: (A) being stranded in the grasslands; (B) how you're going to protect your photographic equipment, find interesting objects and scenes to photograph, and have adequate lighting for the photography; (C) the dinner party."

\section{Procedure}

Participants completed the study using computers in a university computer lab. In the incidental learning phase, participants either first imagined a narrative and then proceeded to the rating task or proceeded directly to the pleasantness rating task.

During the rating task, all participants rated 150 words on their relevance to the narrative or their degree of pleasantness. Following this, participants directly 
proceeded to the retention phase, during which they completed a five-minute distraction task (i.e., calculating moderately difficult math problems). Finally, participants completed a surprise free recall memory test.

\section{Results}

\section{True Recall}

A univariate general linear model (GLM) analysis on the mean number of correctly recalled words (i.e., true recall) was conducted. The factor was processing task (i.e., experimental condition). There was a significant main effect of processing task, $F(3,216)=4.08, p=.008, \eta \mathrm{P}^{2}=.05$.

The survival processing hypothesis predicts that the survival processing task should produce greater true recall than the non-survival tasks. The planning hypothesis predicts that the planning tasks should produce similar amounts of true recall relative to each other, and greater true recall than the pleasantness task.

The following planned comparisons tested these predictions using Welch's $t$. There was a survival processing advantage on true recall relative to non-survival planning (relative to grasslands photo, $t(112)=-2.14, p<.04, d=.40,95 \%$ CI [$7.68,-.29$ ], and dinner party, $t(112)=4.40, p<.001, d=.82,95 \%$ CI [4.03, 10.61]). The survival processing effect did not replicate when compared to the standard pleasantness processing task, $t(112)=1.32, p=.19$. See Figure 1 .

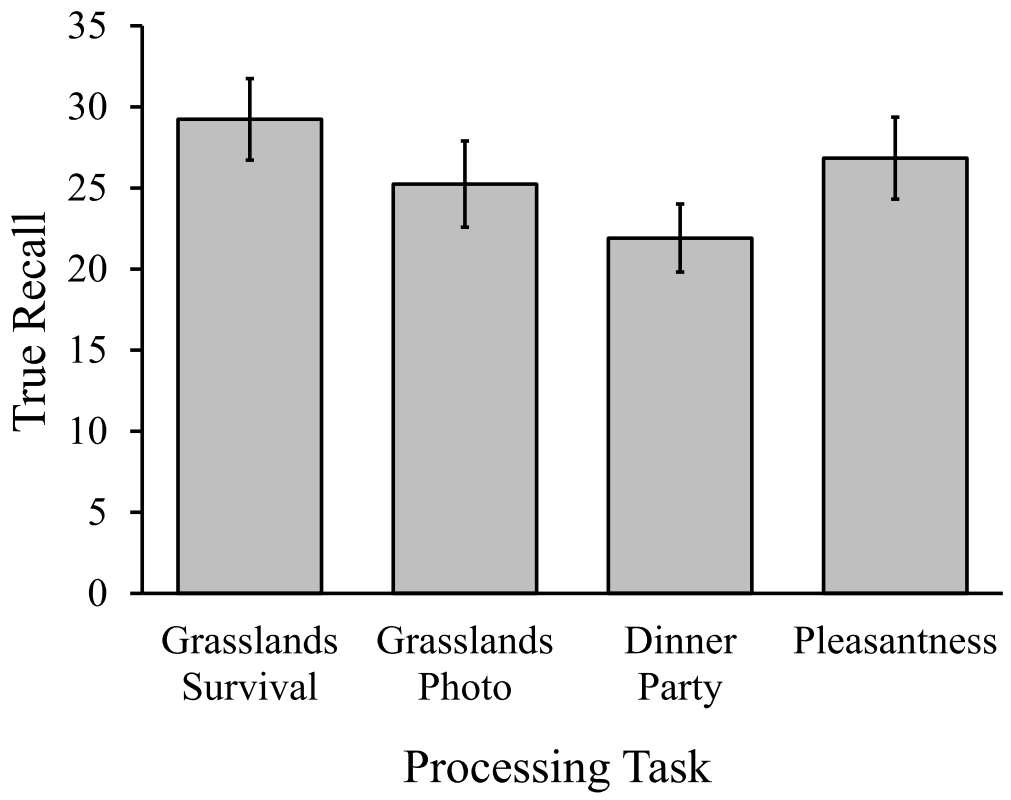

Figure 1. Mean number of words correctly recalled. Error bars $=95 \% \mathrm{CI}$. 


\section{False Recall}

A univariate GLM analysis was conducted on the mean number of intrusions (i.e., false recall). The factor was processing task. There was a main effect of processing task, $F(3,216)=5.43, p=.001, \eta \mathrm{P}^{2}=.07$.

The survival processing hypothesis predicts that the survival processing task should produce greater false recall than the non-survival tasks. The planning hypothesis predicts that the planning tasks should produce similar amounts of false recall relative to each other, and greater false recall than the pleasantness task.

The following planned comparisons tested these predictions using Welch's $t$. There was a survival processing effect on false recall relative to pleasantness processing, $t(75.68)=3.85, p<.001, d=.73,95 \% \mathrm{CI}[1.24,3.81]$. That is, greater false recall occurred after survival processing than after pleasantness processing. Additionally, greater false recall occurred after the other planning tasks relative to the pleasantness task. That is, greater false recall occurred after grasslands photo processing than after pleasantness processing, $t(91.94)=2.31, p=.02, d=.54$, $95 \%$ CI $[.17,2.24]$. Greater false recall occurred after dinner party processing than after pleasantness processing, $t(104.76)=3.87, p<.001, d=.72,95 \% \mathrm{CI}$ $[.84,2.61]$. See Figure 2.

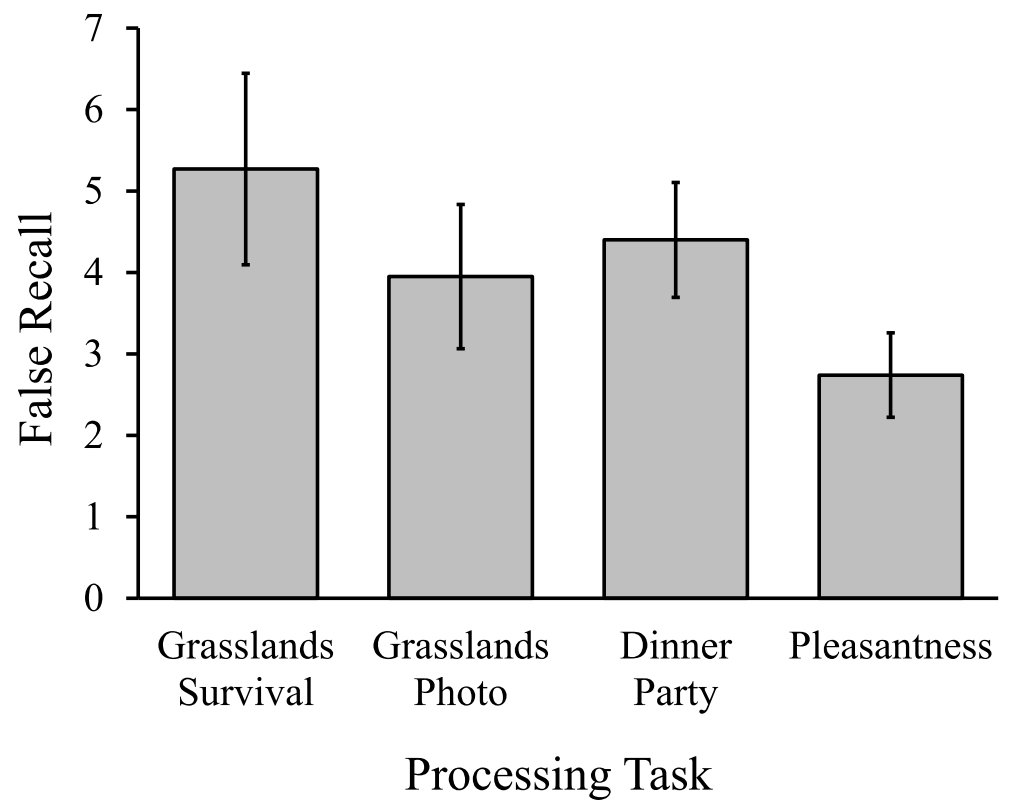

Figure 2. Mean number of words falsely recalled. Error bars $=95 \% \mathrm{CI}$. 


\section{Conclusions}

The survival processing advantage in recall memory (i.e., true recall) appears to depend not simply on planning or invoking a grasslands location but on invoking survival in that location. Additionally, survival processing increased false recall relative to the pleasantness condition, suggesting that survival processing may not produce superior memory overall. Similarly, the other contextually-rich planning tasks produced elevated false recall relative to the pleasantness task. This suggests that the false recall associated with survival processing may be at least partially explained by the presence of planning or rich contextual information.

\section{References}

[1] Nairne JS, Pandeirada JNS, Thompson SR. Adaptive memory: The comparative value of survival processing. Psychological Science. 2008; 19(2):176-80. https://doi.org/10.1111\%2Fj.1467-9280.2008.02064.x

[2] Otgaar H, Howe ML, Smeets T, Raymaekers L, van Beers J. Memory errors in adaptive recollections. What is adaptive about adaptive memory. 2014:201-14.

[3] Klein, SB, Robertson TE, Delton, AW. Facing the future: Memory as an evolved system for planning future acts. Memory \& Cognition, 2010 Jan 1;38(1):13-22. doi: 10.3758/MC.38.1.13

[4] Klein SB, Robertson TE, Delton AW. The future-orientation of memory: Planning as a key component mediating the high levels of recall found with survival processing. Memory. 2011 Feb 1;19(2):121-39. doi: 10.1080/09658211.2010.537827 